\title{
Probabilistic wind speed forecasting using Bayesian model averaging with truncated normal components
}

\author{
SÁNDOR BARAN \\ Faculty of Informatics, University of Debrecen \\ Kassai út 26, H-4028 Debrecen, Hungary \\ and \\ Institute of Applied Mathematics, University of Heidelberg \\ Im Neuenheimer Feld 294, D-69120 Heidelberg, Germany
}

\begin{abstract}
Bayesian model averaging (BMA) is a statistical method for post-processing forecast ensembles of atmospheric variables, obtained from multiple runs of numerical weather prediction models, in order to create calibrated predictive probability density functions (PDFs). The BMA predictive PDF of the future weather quantity is the mixture of the individual PDFs corresponding to the ensemble members and the weights and model parameters are estimated using ensemble members and validating observation from a given training period.

In the present paper we introduce a BMA model for calibrating wind speed forecasts, where the components PDFs follow truncated normal distribution with cut-off at zero, and apply it to the ALADIN-HUNEPS ensemble of the Hungarian Meteorological Service. Three parameter estimation methods are proposed and each of the corresponding models outperforms the traditional gamma BMA model both in calibration and in accuracy of predictions. Moreover, since here the maximum likelihood estimation of the parameters does not require numerical optimization, modelling can be performed much faster than in case of gamma mixtures.

Key words: Bayesian model averaging, continuous ranked probability score, ensemble calibration, truncated normal distribution.
\end{abstract}

\section{Introduction}

The most important aim of weather forecasting is to give a robust and reliable prediction of the future state of the atmosphere based on observational data, prior forecasts and mathe- 
matical models describing the dynamical and physical behaviour of the atmosphere. These models consists of sets of hydro-thermodynamic non-linear partial differential equations of the atmosphere and its coupled systems (like surface or oceans for instance) and have only numerical solutions. The difficulty with these numerical weather prediction models is that since the atmosphere has a chaotic character the solutions strongly depend on the initial conditions and also on other uncertainties related to the numerical weather prediction process. Therefore, the results of such models are never fully accurate. A possible solution is to run the model with different initial conditions (since the uncertainties in the initial conditions are one of the most important sources of uncertainty) and produce an ensemble of forecasts. Using a forecast ensemble one can estimate the probability distribution of future weather variables which allows probabilistic weather forecasting (Gneiting and Raftery, 2005), where not only the future atmospheric states are predicted, but also the related uncertainty information. The ensemble prediction method was proposed by Leith (1974) and since its first operational implementation (Buizza et al., 1993; Toth and Kalnay, 1997) it has become a widely used technique all over the world and the users understand more and more its merits and economic value as well. However, although e.g. the ensemble mean on average yields better forecasts of a meteorological quantity than any of the individual ensemble members, it is often the case that the ensemble is under-dispersive and in this way, uncalibrated (Buizza et al., 2005), so that calibration is needed to account for this deficiency.

The Bayesian model averaging (BMA) method for post-processing ensembles in order to calibrate them was introduced by Raftery et al. (2005). The basic idea of BMA is that for each member of the ensemble forecast there is a corresponding conditional probability density function (PDF) that can be interpreted as the conditional PDF of the future weather quantity provided the considered forecast is the best one. Then the BMA predictive PDF of the future weather quantity is the weighted sum of the individual PDFs corresponding to the ensemble members and the weights are based on the relative performance of the ensemble members during a given training period. In this way BMA is a special, fixed parameter version of dynamic model averaging method developed by Raftery et al. (2010). The weight parameters and the parameters of the individual PDFs are estimated using linear regression and maximum likelihood (ML) method, where the maximum of the likelihood function is found by EM algorithm. In practice, the performance of the individual ensemble members should have a clear characteristic (and not a random one) or if it is not the case this fact should be taken into account at the calibration process (see e.g. Fraley et al., 2010). In Raftery et al. (2005) the BMA method was successfully applied to obtain 48 hour forecasts of surface temperature and sea level pressure in the North American Pacific Northwest based on the 5 members of the University of Washington Mesoscale Ensemble (Grimit and Mass, 2002). These weather quantities can be modeled by normal distributions, so the predictive PDF is a Gaussian mixture. Later, Sloughter et al. (2007) developed a discrete-continuous BMA model for precipitation forecasting, where the discrete part corresponds to the event of no precipitation, while the cubic root of the precipitation amount (if it is positive) is 
modeled by a gamma distribution. In Sloughter et al. (2010) the BMA method was used for wind speed forecasting and the component PDFs follow gamma distributions, while using von Mises distribution to model angular data, Bao et al. (2010) introduced a BMA scheme to predict surface wind direction. Finally, Sloughter et al. (2012) described a BMA model for wind vector forecasting, where wind vectors are modeled using bivariate normal distribution.

The bivariate normal model for wind vectors is also used in the ensemble model output statistics (EMOS) method for post-processing ensemble forecasts (Schuhen et al., 2012). The EMOS, introduced by Gneiting et al. (2005) for calibrating ensemble forecasts following normal distribution (sea level pressure, temperature), produces a single normal PDF, where the mean and the variance depends on the ensemble members. However, the method can be extended to truncated normal distribution (Thorarinsdottir and Gneiting, 2010), too, and in this way it can be used for calibrating wind speed data.

In the present paper we develop a BMA model for wind speed forecasting where the component PDFs, similarly to the EMOS PDF of Thorarinsdottir and Gneiting (2010), follow truncated normal distribution. The advantage of this model to the gamma model of Sloughter et al. (2010) is that for parameter estimation the truncated data EM algorithm for Gaussian mixture models (Lee and Scott, 2012) can be used which works with closed formulae both in expectation (E) and in maximization (M) steps. In this way the estimation of parameters is much faster, which is a key issue in operational applications.

We test our model on the ensemble forecasts of wind speed produced by the operational Limited Area Model Ensemble Prediction System (LAMEPS) of the Hungarian Meteorological Service (HMS) called ALADIN-HUNEPS (Hágel, 2010; Horánvi et al., 2011) and compare the results obtained with the corresponding results of Baran et al. (2013a) where for calibration the BMA gamma model of Sloughter et al. (2010) was used.

\section{Data}

The ALADIN-HUNEPS system of the HMS covers a large part of Continental Europe with a horizontal resolution of $12 \mathrm{~km}$ and it is obtained by dynamical downscaling (by the ALADIN limited area model) of the global ARPEGE based PEARP system of Météo France (Horányi et al., 2006; Descamps et al., 2009). The ensemble consists of 11 members, 10 initialized from perturbed initial conditions and one control member from the unperturbed analysis, implying that the ensemble contains groups of exchangeable forecasts. The data base contains 11 member ensembles of 42 hour forecasts for 10 meter wind speed (given in m/s) for 10 major cities in Hungary (Miskolc, Szombathely, Győr, Budapest, Debrecen, Nyíregyháza, Nagykanizsa, Pécs, Kecskemét, Szeged) produced by the ALADIN-HUNEPS system of the HMS, together with the corresponding validating observations for the period between October 1, 2010 and March 25, 2011 (176 days, or 1760 data points). The forecasts are initialized at 18 UTC. The data set is fairly complete since there are only two days 


\section{Verification Rank Histogram}

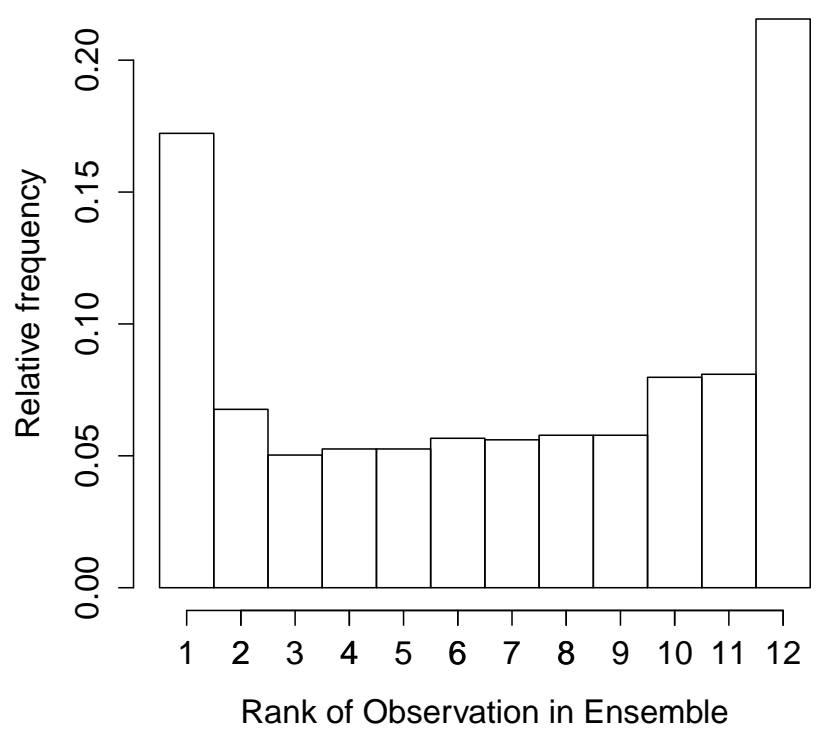

Figure 1: Verification rank histogram of the 11-member ALADIN-HUNEPS ensemble. Period: October 1, 2010 - March 25, 2011.

(18.10.2010 and 15.02.2011) where three ensemble members are missing for all sites and one day (20.11.2010) when no forecasts are available.

Figure 1 shows the verification rank histogram of the raw ensemble. This is the histogram of ranks of validating observations with respect to the corresponding ensemble forecasts computed from the ranks at all stations and over the whole verification period (see e.g. Wilks, 2011, Section 7.7.2). This histogram is far from the desired uniform distribution as in many cases the ensemble members either underestimate or overestimate the validating observations (the ensemble range contains the observed wind speed in $61.21 \%$ of the cases, while its nominal value equals $10 / 12$, i.e $83.33 \%$ ). Hence, the ensemble is under-dispersive and in this way it is uncalibrated. Therefore, statistical post-processing is required to improve the forecasted probability density function.

\section{Methods}

\subsection{Bayesian model averaging}

Let $f_{1}, f_{2}, \ldots, f_{M}$ denote the ensemble forecast of a certain weather quantity $X$ for a given location and time. In BMA for ensemble forecasting (Raftery et al., 2005) to each ensemble member $f_{k}$ corresponds a component PDF $g_{k}\left(x \mid f_{k}, \theta_{k}\right)$, where $\theta_{k}$ is a parameter to be 
estimated. The BMA predictive PDF of $X$ is

$$
p\left(x \mid f_{1}, \ldots, f_{M} ; \theta_{1}, \ldots, \theta_{M}\right):=\sum_{k=1}^{M} \omega_{k} g_{k}\left(x \mid f_{k}, \theta_{k}\right),
$$

where the weight $\omega_{k}$ is connected to the relative performance of the ensemble member $f_{k}$ during the training period. Obviously, the weights form a probability distribution and in this way they are nonnegative and $\sum_{k=1}^{M} \omega_{k}=1$.

BMA model (3.1) is valid only in the cases when the sources of the ensemble members are clearly distinguishable, as for the University of Washington mesoscale ensemble (Eckel and Mass, 2005). However, most of the currently used ensemble prediction systems produce ensembles where some ensemble members are statistically indistinguishable. Usually, these exchangeable ensemble members are obtained with the help of perturbations of the initial conditions, which is the case for the 51 member European Centre for Medium-Range Weather Forecasts ensemble (Leutbecher and Palmer, 2008) or for the ALADIN-HUNEPS ensemble described in Section 2.

Suppose we have $M$ ensemble members divided into $m$ exchangeable groups, where the $k$ th group contains $M_{k} \geq 1$ ensemble members, so $\sum_{k=1}^{m} M_{k}=M$. Further, denote by $f_{k, \ell}$ the $\ell$ th member of the $k$ th group. For this situation Fraley et al. (2010) suggested to use model

$$
p\left(x \mid f_{1,1}, \ldots, f_{1, M_{1}}, \ldots, f_{m, 1}, \ldots, f_{m, M_{m}} ; \theta_{1}, \ldots, \theta_{m}\right):=\sum_{k=1}^{m} \sum_{\ell=1}^{M_{k}} \omega_{k} g_{k}\left(x \mid f_{k, \ell}, \theta_{k}\right),
$$

where ensemble members within a given group have the same weights, PDFs and parameters.

To simplify notations we give the results and formulae of this section for model (3.1), but their generalization to model (3.2) is rather straightforward.

\subsection{Truncated normal model}

As it was mentioned in the Introduction, for weather variables such as temperature, pressure or wind vectors BMA models with normal component PDFs can be fit reasonably well. However, for modeling wind speed, which can take only nonnegative values, a skewed distribution is required. A popular candidate is the Weibull distribution (see e.g. Justus et al., 1978), while Sloughter et al. (2010) considers a BMA model based on gamma distribution. Here we follow the ideas of Gneiting et al. (2006) and Thorarinsdottir and Gneiting (2010) and for wind speed modeling we employ a truncated normal distribution with a cut-off at zero $\mathcal{N}^{0}\left(\mu, \sigma^{2}\right)$ with $\mathrm{PDF}$

$$
g(x \mid \mu, \sigma):=\frac{\frac{1}{\sigma} \varphi((x-\mu) / \sigma)}{\Phi(\mu / \sigma)}, \quad x \geq 0, \quad \text { and } \quad g(x \mid \mu, \sigma):=0, \quad \text { otherwise }
$$


where $\varphi$ and $\Phi$ denote the PDF and the cumulative distribution function (CDF) of the standard normal distribution, respectively. The mean $\kappa$ and variance $\varrho^{2}$ of $\mathcal{N}^{0}\left(\mu, \sigma^{2}\right)$ are

$$
\kappa=\mu+\frac{\sigma \varphi(\mu / \sigma)}{\Phi(\mu / \sigma)} \quad \text { and } \quad \varrho^{2}=\sigma^{2}\left(1-\frac{\mu}{\sigma} \frac{\varphi(\mu / \sigma)}{\Phi(\mu / \sigma)}-\left(\frac{\varphi(\mu / \sigma)}{\Phi(\mu / \sigma)}\right)^{2}\right)
$$

respectively.

We assume that location $\mu$ is a linear function of the forecasted wind speed that leads to BMA mixture model

$$
p\left(x \mid f_{1}, \ldots, f_{M} ; \alpha_{1}, \ldots, \alpha_{M} ; \beta_{1}, \ldots, \beta_{M} ; \sigma_{1}, \ldots, \sigma_{M}\right):=\sum_{k=1}^{M} \omega_{k} g\left(x \mid \alpha_{k}+\beta_{k} f_{k}, \sigma_{k}\right),
$$

where $g$ is the PDF defined by (3.3). To simplify the model, in what follows we also assume $\sigma_{1}=\sigma_{2}=\ldots=\sigma_{M}=: \sigma$. This reduces the number of parameters and makes computations easier. Furthermore, the BMA gamma model of Sloughter et al. (2010), used as a reference, also operates with this restriction.

\subsection{Continuous ranked probability score}

Continuous ranked probability score (CRPS) is the most popular scoring rule for evaluating density forecasts (Gneiting and Raftery, 2007; Wilks, 2011). Given a CDF F(y) and a real number $x$, the CRPS is defined as

$$
\operatorname{crps}(F, x):=\int_{-\infty}^{\infty}\left(F(y)-\mathbb{1}_{\{y \geq x\}}\right)^{2} \mathrm{~d} y=\mathrm{E}|X-x|-\frac{1}{2} \mathrm{E}\left|X-X^{\prime}\right|,
$$

where $X$ and $X^{\prime}$ are independent random variables with CDF $F$ and finite first moment. CRPS is a proper scoring rule which is negatively oriented, i.e. the smaller the better, and it can be reported in the same unit as the observation.

Now, if $X \sim \mathcal{N}^{0}\left(\mu, \sigma^{2}\right)$ then short calculation shows

$$
S_{1}(x, \mu, \sigma):=\mathrm{E}|X-x|=[\Phi(\mu / \sigma)]^{-1}\left[A\left(x-\mu, \sigma^{2}\right)+(x-\mu)(\Phi(\mu / \sigma)-1)-\sigma \varphi(\mu / \sigma)\right],
$$

where $A\left(\mu, \sigma^{2}\right):=\mathrm{E}|Y|$ with $Y \sim \mathcal{N}\left(\mu, \sigma^{2}\right)$, that is

$$
A\left(\mu, \sigma^{2}\right)=\mu(2 \Phi(\mu / \sigma)-1)+2 \sigma \varphi(\mu / \sigma) .
$$

Further, if $X_{1} \sim \mathcal{N}^{0}\left(\mu_{1}, \sigma_{1}^{2}\right)$ and $X_{2} \sim \mathcal{N}^{0}\left(\mu_{2}, \sigma_{2}^{2}\right)$ are independent, then the PDF of $\left|X_{1}-X_{2}\right|$ is

$$
f_{\left|X_{1}-X_{2}\right|}(x)=\left[\sigma_{d} \Phi\left(\frac{\mu_{1}}{\sigma_{1}}\right) \Phi\left(\frac{\mu_{2}}{\sigma_{2}}\right)\right]^{-1}\left[\varphi\left(\frac{x-\mu_{d}}{\sigma_{d}}\right) \Phi\left(\varrho_{d}-\frac{\sigma_{2} x}{\sigma_{1} \sigma_{d}}\right)+\varphi\left(\frac{x+\mu_{d}}{\sigma_{d}}\right) \Phi\left(\varrho_{d}-\frac{\sigma_{1} x}{\sigma_{2} \sigma_{d}}\right)\right]
$$


where

$$
\mu_{d}:=\mu_{1}-\mu_{2}, \quad \sigma_{d}:=\sqrt{\sigma_{1}^{2}+\sigma_{2}^{2}} \quad \text { and } \quad \varrho_{d}:=\frac{\mu_{1} \sigma_{2}^{2}+\mu_{2} \sigma_{1}^{2}}{\sigma_{1} \sigma_{2} \sigma_{d}}
$$

Hence,

$$
\begin{aligned}
S_{2}\left(\mu_{1}, \mu_{2}, \sigma_{1}, \sigma_{2}\right):=\mathrm{E}\left|X_{1}-X_{2}\right|= & {\left[\Phi\left(\mu_{1} / \sigma_{1}\right) \Phi\left(\mu_{2} / \sigma_{2}\right)\right]^{-1} } \\
& \times\left[A\left(\mu_{1}-\mu_{2}, \sigma_{1}^{2}+\sigma_{2}^{2}\right)-\sqrt{\sigma_{1}^{2}+\sigma_{2}^{2}} C\left(\mu_{1}, \mu_{2}, \sigma_{1}, \sigma_{2}\right)\right],
\end{aligned}
$$

where the correction term

$$
C\left(\mu_{1}, \mu_{2}, \sigma_{1}, \sigma_{2}\right):=\int_{0}^{\infty} x\left[\varphi\left(x-\frac{\mu_{d}}{\sigma_{d}}\right) \Phi\left(\frac{\sigma_{2}}{\sigma_{1}} x-\varrho_{d}\right)+\varphi\left(x+\frac{\mu_{d}}{\sigma_{d}}\right) \Phi\left(\frac{\sigma_{1}}{\sigma_{2}} x-\varrho_{d}\right)\right] \mathrm{d} x
$$

can only be evaluated numerically. Now, using (3.6) one can easily obtain the CRPS corresponding to the $\mathrm{CDF} \mathcal{P}$ of mixture model (3.5), namely

$$
\operatorname{crps}(\mathcal{P}, x)=\sum_{k=1}^{M} \omega_{k} S_{1}\left(x, \alpha_{k}+\beta_{k} f_{k}, \sigma_{k}\right)-\frac{1}{2} \sum_{k=1}^{M} \sum_{\ell=1}^{M} \omega_{k} \omega_{\ell} S_{2}\left(\alpha_{k}+\beta_{k} f_{k}, \alpha_{\ell}+\beta_{\ell} f_{\ell}, \sigma_{k}, \sigma_{\ell}\right) .
$$

\subsection{Parameter estimation}

Parameters $\alpha_{k}, \beta_{k}, \omega_{k}, k=1,2, \ldots M$, and $\sigma$ are estimated using training data consisting of ensemble members and verification observations from the preceding $n$ days (training period). In what follows, $f_{k, s, t}$ denotes the $k$ th ensemble member for location $s \in \mathcal{S}$ and time $t \in \mathcal{T}$ and by $x_{s, t}$ we denote the corresponding validating observation. We consider three approaches which mainly differ in estimation of the parameters $\alpha_{k}, \beta_{k}$ of location.

\subsubsection{Naive approach}

Similarly to the normal BMA model of Raftery et al. (2005) we estimate location parameters $\alpha_{k}$ and $\beta_{k}$ with a linear regression of $x_{s, t}$ on $f_{k, s, t}$ over the time points in the training period. We call this approach naive since it does not take into account that for truncated normal distribution $\mathcal{N}^{0}\left(\mu, \sigma^{2}\right)$ location $\mu$ does not equal to the mean $\kappa$. However, one should remark that with the increase of $\mu$ the correction term in (3.4) decreases in an exponential rate.

To estimate weights $\omega_{k}, k=1,2, \ldots, M$, and scale parameter $\sigma$ maximum likelihood method is applied using again training data. Under the assumption of independence of forecast errors in space and time the log-likelihood function corresponding to model (3.5) equals

$$
\ell\left(\omega_{1}, \ldots, \omega_{M} ; \sigma\right)=\sum_{s, t} \log \left[\sum_{k=1}^{M} \omega_{k} g\left(x_{s, t} \mid \alpha_{k}+\beta_{k} f_{k, s, t}, \sigma\right)\right]
$$


where the first summation is over all locations $s \in \mathcal{S}$ and time points $t$ from the training period containing $N$ terms ( $N$ distinct values of $(s, t)$ ).

The log-likelihood function (3.7) is too complicated to be maximized analytically, so we find its maximum using the truncated data EM algorithm of Lee and Scott (2012). Similarly to the traditional EM algorithm for mixtures (Dempster et al., 1977; McLachlan and Krishnan, 1997) we introduce latent indicator variables $z_{k, s, t}$ taking values one or zero according as whether $x_{s, t}$ comes from the $k$ th component PDF or not. The complete data log-likelihood corresponding to observations and indicator variables equals

$$
\ell_{C}\left(\omega_{1}, \ldots, \omega_{M} ; \sigma\right)=\sum_{s, t} \sum_{k=1}^{M} z_{k, s, t}\left[\log \left(\omega_{k}\right)+\log \left(g\left(x_{s, t} \mid \alpha_{k}+\beta_{k} f_{k, s, t}, \sigma\right)\right)\right] .
$$

The EM algorithm alternates between an expectation (E) step and a maximization (M) step until convergence. It starts with initial values $\omega_{k}^{(0)}, k=1,2, \ldots, M$, and $\sigma^{(0)}$ of the parameters. In the E step the latent variables are estimated using the conditional expectation of the complete log-likelihood on the observed data, while in the $\mathrm{M}$ step the parameter estimates are updated by maximizing $\ell_{C}$ with the current values of the latent variables plugged in.

For the truncated normal mixture model given by (3.3) and (3.5) the E step is,

$$
z_{k, s, t}^{(j+1)}:=\frac{\omega_{k}^{(j)} g\left(x_{s, t} \mid \alpha_{k}+\beta_{k} f_{k, s, t}, \sigma^{(j)}\right)}{\sum_{i=1}^{M} \omega_{i}^{(j)} g\left(x_{s, t} \mid \alpha_{i}+\beta_{i} f_{i, s, t}, \sigma^{(j)}\right)},
$$

where the superscript refers to the actual iteration. The first part of the $\mathrm{M}$ step is obviously

$$
\omega_{k}^{(j+1)}:=\frac{1}{N} \sum_{s, t} z_{k, s, t}^{(j+1)}
$$

while the second part can be derived from equation $\frac{\partial \ell_{C}}{\partial \sigma}=0$. However, in our case this equation is nonlinear and since it cannot be solved for $\sigma$, we suggest iteration step

$$
\begin{aligned}
\sigma^{2(j+1)}=\frac{1}{N} & \sum_{s, t} \sum_{k=1}^{M} z_{k, s, t}^{(j+1)}\left(x_{s, t}-\alpha_{k}-\beta_{k} f_{k, s, t}\right)^{2} \\
& +\frac{\sigma^{(j)}}{N} \sum_{s, t} \sum_{k=1}^{M} z_{k, s, t}^{(j+1)}\left(\alpha_{k}+\beta_{k} f_{k, s, t}\right) \frac{\varphi\left(\left(\alpha_{k}+\beta_{k} f_{k, s, t}\right) / \sigma^{(j)}\right)}{\Phi\left(\left(\alpha_{k}+\beta_{k} f_{k, s, t}\right) / \sigma^{(j)}\right)} .
\end{aligned}
$$

Observe that the EM algorithm presented here differs from the corresponding algorithm of Raftery et al. (2005) only in the second term of (3.11).

\subsubsection{Mean corrected approach}

In this approach we assume that the means of the component PDFs are also linear functions of form $a_{k}+b_{k} f_{k, s, t}$ of the forecasted wind speed and the linear regression of $x_{s, t}$ on $f_{k, s, t}$ 
is now used to estimate $a_{k}$ and $b_{k}, k=1,2, \ldots, M$. Instead of (3.8) we consider the complete data log-likelihood

$$
\ell_{C}\left(\omega_{1}, \ldots, \omega_{M}, \sigma\right)=\sum_{s, t} \sum_{k=1}^{M} z_{k, s, t}\left[\log \left(\omega_{k}\right)+\log \left(g\left(x_{s, t} \mid \mu_{k, s, t}, \sigma\right)\right)\right]
$$

where the initial guess for the location parameter $\mu_{k, s, t}$ of the $k$ th component PDF at $(s, t)$ is $\mu_{k, s, t}^{(0)}:=a_{k}+b_{k} f_{k, s, t}$.

Now, E step (3.9) is replaced by

$$
z_{k, s, t}^{(j+1)}:=\frac{\omega_{k}^{(j)} g\left(x_{s, t} \mid \mu_{k, s, t}^{(j)}, \sigma^{(j)}\right)}{\sum_{i=1}^{M} \omega_{i}^{(j)} g\left(x_{s, t} \mid \mu_{i, s, t}^{(j)}, \sigma^{(j)}\right)}
$$

the first part (3.10) of the M step remains valid, while

$$
\mu_{k, s, t}^{(j+1)}:=\mu_{k, s, t}^{(0)}-\sigma^{(j)} \frac{\varphi\left(\mu_{k, s, t}^{(j)} / \sigma^{(j)}\right)}{\Phi\left(\mu_{k, s, t}^{(j)} / \sigma^{(j)}\right)}
$$

and

$$
\sigma^{2(j+1)}=\frac{1}{N} \sum_{s, t} \sum_{k=1}^{M} z_{k, s, t}^{(j+1)}\left(x_{s, t}-\mu_{k, s, t}^{(j+1)}\right)^{2}+\frac{\sigma^{(j)}}{N} \sum_{s, t} \sum_{k=1}^{M} z_{k, s, t}^{(j+1)} \mu_{k, s, t}^{(j+1)} \frac{\varphi\left(\mu_{k, s, t}^{(j+1)} / \sigma^{(j)}\right)}{\Phi\left(\mu_{k, s, t}^{(j+1)} / \sigma^{(j)}\right)}
$$

substitute iteration step (3.11). Observe that (3.13) is responsible for the mean correction in the first term of (3.4). Finally, after the EM algorithm stops, parameters $\alpha_{k}$ and $\beta_{k}$ are estimated with a linear regression of the final value of $\mu_{k, s, t}$ on $f_{k, s, t}$.

\subsubsection{Full maximum likelihood estimation}

In the previous two cases the estimates of location parameters $\alpha_{k}$ and $\beta_{k}, k=1,2, \ldots, M$, are obtained separately from the weights and the scale parameter. Here we present a method where all parameters are estimated with ML method using the complete data log-likelihood defined by (3.8). Obviously, in this case $\ell_{C}$ is considered as a function of $\alpha_{k}, \beta_{k}, \omega_{k}, k=$ $1,2, \ldots, M$, and $\sigma$. The initial guesses $\alpha_{k}^{(0)}$ and $\beta_{k}^{(0)}$ for these parameters are the corresponding mean coefficients obtained from regressing of $x_{s, t}$ on $f_{k, s, t}$ over the time points in the training period. In this way the initial value $\mu_{k, s, t}^{(0)}:=\alpha_{k}^{(0)}+\beta_{k}^{(0)} f_{k, s, t}$ of the location parameter is exactly the estimated mean of the $k$ th component PDF.

The E step remains the same as in the mean corrected approach, that is estimate $z_{k, s, t}^{(j+1)}$ of the latent variable $z_{k, s, t}$ is given by (3.12). The first part (3.10) of the M step remains 
valid again, while equations $\frac{\partial \ell_{C}}{\partial \alpha_{k}}$ and $\frac{\partial \ell_{C}}{\partial \beta_{k}}$ result in iterations

$$
\begin{aligned}
\alpha_{k}^{(j+1)} & :=\left[\sum_{s, t} z_{k, s, t}^{(j+1)}\right]^{-1}\left[\sum_{s, t} z_{k, s, t}^{(j+1)}\left(\left(x_{s, t}-\beta_{k}^{(j)} f_{k, s, t}\right)-\sigma^{(j)} \frac{\varphi\left(\mu_{k, s, t}^{(j)} / \sigma^{(j)}\right)}{\Phi\left(\mu_{k, s, t}^{(j)} / \sigma^{(j)}\right)}\right)\right], \\
\beta_{k}^{(j+1)}: & :\left[\sum_{s, t} z_{k, s, t}^{(j+1)} f_{k, s, t}^{2}\right]^{-1}\left[\sum_{s, t} z_{k, s, t}^{(j+1)} f_{k, s, t}\left(\left(x_{s, t}-\alpha_{k}^{(j+1)}\right)-\sigma^{(j)} \frac{\varphi\left(\mu_{k, s, t}^{(j)} / \sigma^{(j)}\right)}{\Phi\left(\mu_{k, s, t}^{(j)} / \sigma^{(j)}\right)}\right)\right] .
\end{aligned}
$$

Mean correction (3.13) takes form

$$
\mu_{k, s, t}^{(j+1)}:=\mu_{k, s, t}^{(0)}-\sigma^{(j)} \frac{\varphi\left(\left(\alpha_{k}^{(j+1)}+\beta_{k}^{(j+1)} f_{k, s, t}\right) / \sigma^{(j)}\right)}{\Phi\left(\left(\alpha_{k}^{(j+1)}+\beta_{k}^{(j+1)} f_{k, s, t}\right) / \sigma^{(j)}\right)},
$$

while the estimate of variance is updated using (3.14).

\section{Results}

As was mentioned in the Introduction, the performance of BMA model (3.5) is tested on the ALADIN-HUNEPS ensemble of HMS. We consider all three parameter estimating methods of Subsection 3.4 and use the same data base as in Baran et al. (2013a), where the authors calibrated the raw ensemble with the help of the BMA gamma model of Sloughter et al. (2010) considering a training period of 28 calendar days. Here we apply the same training period length which allows direct comparison of the two BMA methods. In this way ensemble members, validating observations and BMA models are available for 146 calendar days (on 20.11.2010 all ensemble members are missing).

\subsection{Models and diagnostics}

Using the ideas of Baran et al. (2013a) we consider two different groupings of ensemble members. In the first case we have two exchangeable groups. One contains the control denoted by $f_{c}$ while in the other are 10 ensemble members corresponding to the different perturbed initial conditions denoted by $f_{p, 1}, \ldots, f_{p, 10}$. This leads us to model

$$
\begin{aligned}
p\left(x \mid f_{c} f_{p, 1}, \ldots, f_{p, 10} ; \alpha_{c}, \alpha_{p} ; \beta_{c}, \beta_{p} ; \sigma\right)= & \omega g\left(x \mid \alpha_{c}+\beta_{c} f_{c}, \sigma\right) \\
& +\frac{1-\omega}{10} \sum_{\ell=1}^{10} g\left(x \mid \alpha_{p}+\beta_{p} f_{p, \ell}, \sigma\right),
\end{aligned}
$$

where $\omega \in[0,1]$, and $g$ is defined by (3.3). 
Two groups, naive

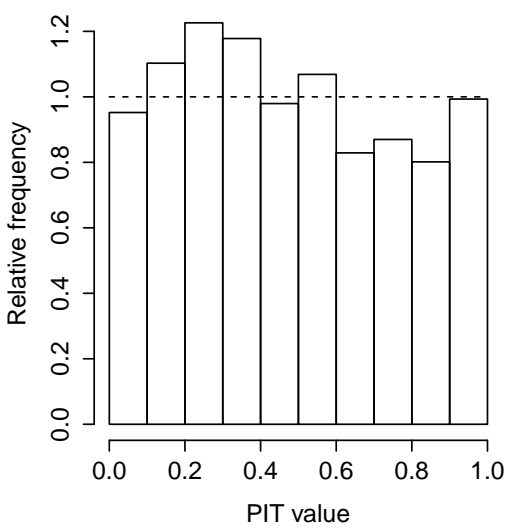

Three groups, naive

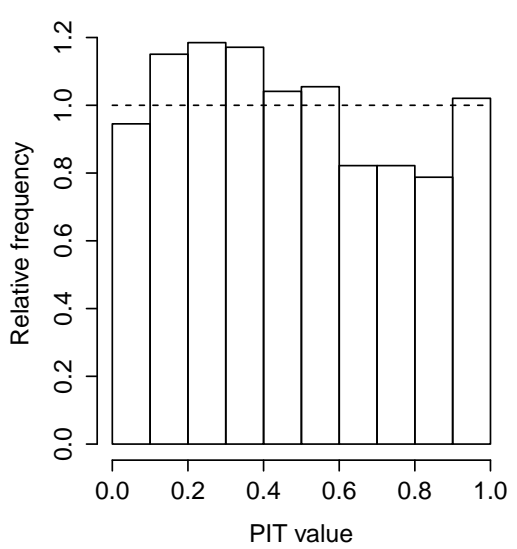

Two groups, mean corrected
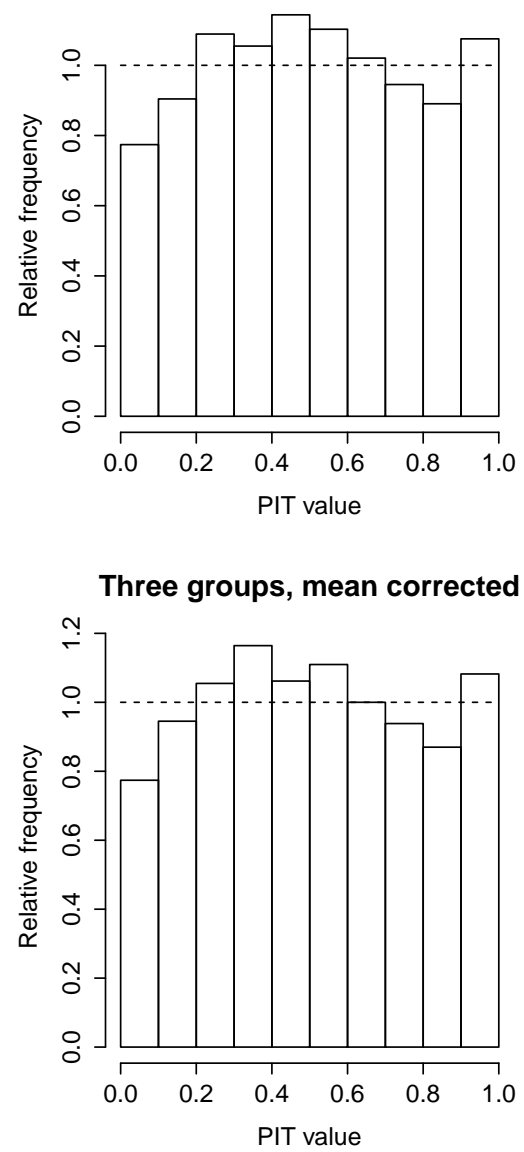

Two groups, full ML

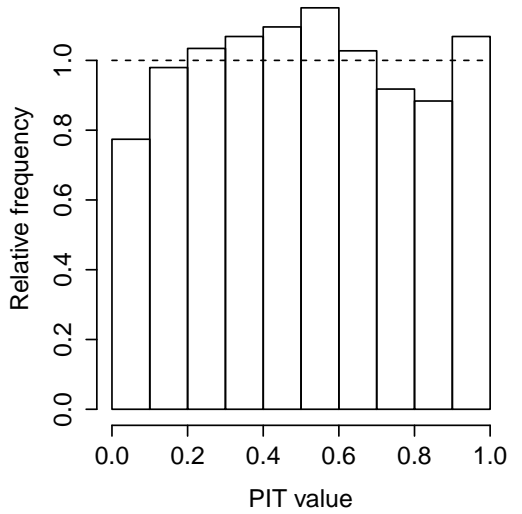

Three groups, full ML

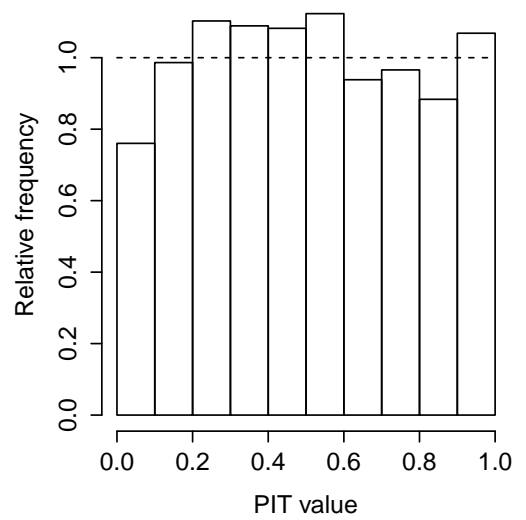

Figure 2: PIT histograms for BMA post-processed forecasts using two-group (4.1) and threegroup (4.2) models.

In the second case the odd and even numbered exchangeable ensemble members form two separate groups $\left\{f_{p, 1}, f_{p, 3}, f_{p, 5}, f_{p, 7}, f_{p, 9}\right\}$ and $\left\{f_{p, 2}, f_{p, 4}, f_{p, 6}, f_{p, 8}, f_{p, 10}\right\}$, respectively, which idea is justified by the method their initial conditions are generated. To get them only five perturbations are calculated and then they are added to (odd numbered members) and subtracted from (even numbered members) the unperturbed initial conditions (Horánvi et al., 2011; Baran et al., 2013a, b). In this way we obtain the following PDF for the forecasted wind speed:

$$
\begin{aligned}
q\left(x \mid f_{c}, f_{p, 1}, \ldots, f_{p, 10} ;\right. & \left.\alpha_{c}, \alpha_{o}, \alpha_{e} ; \beta_{c}, \beta_{o}, \beta_{e} ; \sigma\right)=\omega_{c} g\left(x \mid \alpha_{c}+f_{c} \beta_{c}, \sigma\right) \\
& +\sum_{\ell=1}^{5}\left(\omega_{o} g\left(x \mid \alpha_{o}+\beta_{o} f_{p, 2 \ell-1}, \sigma\right)+\omega_{e} g\left(x \mid \alpha_{e}+\beta_{e} f_{p, 2 \ell}, \sigma\right)\right)
\end{aligned}
$$

where for weights $\omega_{c}, \omega_{o}, \omega_{e} \in[0,1]$ we have $\omega_{c}+5 \omega_{o}+5 \omega_{e}=1$.

In order to check the overall performance of the probabilistic forecasts (based on models 


\begin{tabular}{|l|c|c|c|c|}
\hline & \multicolumn{2}{|c|}{ Truncated normal BMA } & \multirow{2}{*}{ Gamma BMA } \\
\cline { 2 - 4 } & naive & mean corr. & full ML & \\
\hline Two groups & $2.42 \times 10^{-4}$ & $1.79 \times 10^{-2}$ & 0.13 & $2.22 \times 10^{-2}$ \\
Three groups & $1.37 \times 10^{-4}$ & $5.56 \times 10^{-2}$ & 0.18 & $1.87 \times 10^{-2}$ \\
\hline
\end{tabular}

Table 1: Significance levels of Kolmogorov-Smirnov tests for uniformity of PIT values corresponding to two- and three-group models.

(4.1) and (4.2) ) in terms of a probability distribution function, the mean CRPS (the average of the CRPS values of the predictive CDFs and corresponding validating observations taken over all locations and time points considered) and the coverage and average widths of $66.7 \%$ and $90 \%$ central prediction intervals are computed and compared for both BMA methods (truncated normal and gamma) and raw ensemble. In the latter case, the ensemble of forecasts corresponding to a given location and time is considered as a statistical sample, the empirical CDF of the ensemble replaces the predictive CDF and the sample quantiles are calculated according to Hyndman and Fan (1996, Definition 7). We remark that the coverage of a $(1-\alpha) 100 \%, \alpha \in(0,1)$ central prediction interval is the proportion of validating observations located between the lower and upper $\alpha / 2$ quantiles of the predictive distribution. For a calibrated predictive PDF this value should be around $(1-\alpha) 100 \%$. Additionally, the BMA and ensemble medians are considered as point forecasts, which are evaluated with the use of mean absolute errors (MAE) and root mean square errors (RMSE).

\subsection{Verification results of BMA post-processing}

To get a first insight about the calibration of BMA post-processed forecasts we consider probability integral transform (PIT) histograms. The PIT is the value of the predictive cumulative distribution evaluated at the verifying observations (Raftery et al., 2005), which is providing a good measure about the possible improvements of the under-dispersive character of the raw ensemble. The closer the histogram is to the uniform distribution, the better the calibration is. In Figure 2 the PIT histograms corresponding to all three parameter estimating methods and to both BMA models (4.1) and (4.2) are displayed. A comparison to the verification rank histogram of the raw ensemble (see Figure 1) shows that post-processing significantly improves the statistical calibration of the forecasts. Further, on the basis of significance levels of Kolmogorov-Smirnov tests given in Table 1, for both models (4.1) and (4.2) one can accept the uniformity of PIT values corresponding to truncated normal BMA model with full maximum likelihood parameter estimation method. However, one should remark that for the three group model mean corrected parameter estimation also yields acceptable PIT values.

In Table 2 scores for different probabilistic forecasts are given together with the average 


\begin{tabular}{|c|c|c|c|c|c|c|c|c|c|}
\hline \multirow{2}{*}{\multicolumn{3}{|c|}{ Forecast }} & \multirow{2}{*}{$\begin{array}{l}\text { Mean } \\
\text { CRPS }\end{array}$} & \multirow[t]{2}{*}{ MAE } & \multirow[t]{2}{*}{ RMSE } & \multicolumn{2}{|c|}{ Average width } & \multicolumn{2}{|c|}{ Coverage $(\%)$} \\
\hline & & & & & & $66.7 \%$ & $90.0 \%$ & $66.7 \%$ & $90.0 \%$ \\
\hline \multirow{4}{*}{$\begin{array}{l}\text { Two } \\
\text { groups }\end{array}$} & \multirow{3}{*}{$\begin{array}{l}\text { Trunc. } \\
\text { normal } \\
\text { BMA }\end{array}$} & naive & 0.7225 & 1.0631 & 1.3800 & 2.5738 & 4.2850 & 67.81 & 89.79 \\
\hline & & mean c. & 0.7062 & 1.0520 & 1.3784 & 2.6175 & 4.3420 & 69.86 & 90.55 \\
\hline & & full ML & 0 . & 1.0518 & 1.3786 & 2.6029 & 4.3222 & 69.38 & 90.14 \\
\hline & \multicolumn{2}{|c|}{ Gamma BMA } & 0.7577 & 1.0678 & 1.4213 & 2.6359 & 4.5297 & 68.08 & 90.34 \\
\hline \multirow{4}{*}{$\begin{array}{l}\text { Three } \\
\text { groups }\end{array}$} & \multirow{3}{*}{$\begin{array}{l}\text { Trunc. } \\
\text { normal } \\
\text { BMA }\end{array}$} & naive & 0.7213 & 1.0612 & 1.3771 & 2.5645 & 4.2709 & 67.26 & 89.86 \\
\hline & & mean $\mathrm{c}$. & 0.7042 & 1.0480 & 1.3737 & 2.6043 & 4.3195 & 69.38 & 90.34 \\
\hline & & full ML & 0.7044 & 1.0485 & 1.3739 & 2.5948 & 4.3073 & 68.84 & 90.14 \\
\hline & \multicolumn{2}{|c|}{ Gamma BMA } & 0.7556 & 1.0643 & 1.4153 & 2.6153 & 4.4931 & 68.36 & 90.21 \\
\hline \multicolumn{3}{|c|}{ Raw ensemble } & 0.8599 & 1.1215 & 1.4634 & 1.4388 & 2.2001 & 38.70 & 55.14 \\
\hline
\end{tabular}

Table 2: Mean CRPS of probabilistic, MAE and RMSE of median forecasts, average width and coverage of $66.7 \%$ and $90.0 \%$ central prediction intervals.

width and coverage of $66.7 \%$ and $90.0 \%$ central prediction intervals. Verification measures of probabilistic forecasts and point forecasts calculated using truncated normal BMA models (4.1) and (4.2) are compared to the corresponding measures calculated for the raw ensemble and applying gamma BMA post-processing (Baran et al., 2013a). Compared to the raw ensemble all BMA post-processed forecasts show a significant decrease in all there verification scores considered. Further, as the listed CRPS, MAE and RMSE values show, the accuracy of the truncated normal BMA probabilistic and point forecasts is better than the accuracy of the gamma BMA ones.

Concerning calibration, one can observe that the coverage of both BMA central prediction intervals are rather close to the correct coverage for all models considered, while the coverage of the central prediction intervals calculated from the raw ensemble are quite poor. This shows that BMA post-processing greatly improves calibration. Further, the truncated normal BMA models yields slightly sharper predictions than the gamma BMA forecasts and one can also observe that the three-group model slightly outperforms the two-group one.

Finally, we remark that mean correction step (3.15) in full ML parameter estimation method seems essential. Running the algorithm without it (that is $\mu_{k, s, t}^{(j+1)}:=\alpha_{k}^{(j+1)}+$ $\left.\beta_{k}^{(j+1)} f_{k, s, t}\right)$ e.g. for the three group model yields smaller CRPS (0.7024) but larger MAE and RMSE values (1.0499 and 1.3900) and wider central prediction intervals. Moreover, in this case the PIT values do not fit the uniform distribution. 


\section{Discussion}

We introduced a new BMA model for post-processing ensemble forecasts of wind speed providing a predictive PDF which is a mixture of normal distributions truncated from below at zero. The model was tested on the 11 member ALADIN-HUNEPS ensemble of the HMS using two different BMA models. One assumes two groups of exchangeable members (control and forecasts from perturbed initial conditions), while the other considers three (control and forecasts from perturbed initial conditions with positive and negative perturbations). For both models a 28 day training period was used and three types of parameter estimation: a naive and two more sophisticated one with mean correction and full maximum likelihood estimation. The latter resulted PIT values which perfectly fit the uniform distribution both for the two- and for the three-group model. The comparison of the raw ensemble and of the truncated normal BMA forecasts shows that the mean CRPS values of BMA post-processed forecasts are considerably lower than the mean CRPS of the raw ensemble. Furthermore, the MAE and RMSE values of BMA median forecasts are also lower than the MAEs and RMSEs of the ensemble median. The calibration of BMA forecasts is nearly perfect as the coverage of the $66.7 \%$ and $90.0 \%$ prediction intervals are very close to the nominal levels. From the three competing parameter estimation methods the overall performance of the full ML estimation seems to be the best.

Compared to the performance of gamma BMA model of Sloughter et al. (2010) for wind

speed data investigated in Baran et al. (2013a) one can observe that truncated normal BMA post-processing yields slightly lower CRPS, MAE and RMSE values and narrower central prediction intervals. However, the great advantage of the model presented here appears in the speed of parameter estimation. The EM algorithm for estimating the weights and distribution parameters of the truncated normal BMA model uses closed formulae while in case of the gamma BMA model a numerical optimization is required. Running the ensembleBMA package of R (Fraley et al., 2009, 2011) on a PC under a 64bit Window 7 operating system (Intel Core i5-3470 CPU, $3.40 \mathrm{GHz}, 4$ cores, $8 \mathrm{~Gb}$ RAM) the estimation of parameters of the three-group model e.g. for 25.03.2011 using training period of 28 calendar days, for the gamma BMA model took 10.23 seconds, while for the truncated normal with naive, mean corrected and full ML parameter estimation methods, $6.86 \mathrm{~s}, 6.83 \mathrm{~s}$ and $10.41 \mathrm{~s}$, respectively. The difference is more convincing when we perform modeling for all possible 148 calendar days. The corresponding running times were $2716.98 \mathrm{~s}$ for the gamma BMA and $797.89 \mathrm{~s}$, $918.16 \mathrm{~s}$ and $1245.5 \mathrm{~s}$ for the truncated normal BMA models. In this way the truncated normal BMA model outperforms the traditional gamma BMA model both in accuracy and calibration of forecasts and in computation time.

Acknowledgments. Research was supported by the Hungarian Scientific Research Fund under Grant No. OTKA NK101680 and by the TÁMOP-4.2.2.C-11/1/KONV-2012-0001 project. The project has been supported by the European Union, co-financed by the Eu- 
ropean Social Fund. The author is indebted to Tilmann Gneiting and András Horányi for their useful suggestions and remarks and to Máté Mile and Mihály Szücs from the HMS for providing the data.

\section{References}

Bao, L., Gneiting, T., Raftery, A. E., Grimit, E. P. and Guttorp, P. (2010) Bias correction and Bayesian model averaging for ensemble forecasts of surface wind direction. Mon. Wea. Rev. 138, 1811-1821.

Baran, S., Horányi, A., Nemoda, D. (2013a) Statistical post-processing of probabilistic wind speed forecasting in Hungary. Meteorol. Z., to appear.

Baran, S., Horányi, A., Nemoda, D. (2013b) Probabilistic temperature forecasting with statistical calibration in Hungary. arXiv:1303.2133.

Buizza, R., Tribbia, J., Molteni, F. and Palmer, T. (1993) Computation of optimal unstable structures for a numerical weather prediction system. Tellus A 45, 388-407.

Buizza, R., Houtekamer, P. L., Toth, Z., Pellerin, G., Wei, M. and Zhu, Y. (2005) A comparison of the ECMWF, MSC, and NCEP global ensemble prediction systems. Mon. Wea. Rev. 133, 1076-1097.

Dempster, A. P., Laird, N. M. and Rubin, D. B. (1977) Maximum likelihood from incomplete data via the EM algorithm. J. Roy. Statist. Soc. Ser. B 39, 1-39.

Descamps, L., Labadier, C., Joly, A. and Nicolau, J. (2009) Ensemble Prediction at Météo France (poster introduction by Olivier Riviere) 31st EWGLAM and 16th SRNWP meetings, 28th September - 1st October, 2009. Available at: http://srnwp.met.hu/Annual_Meetings/2009/download/sept29/morning/posterpearp.pdf

Eckel, F. A. and Mass, C. F. (2005) Effective mesoscale, short-range ensemble forecasting. Wea. Forecasting 20, 328-350.

Fraley, C., Raftery, A. E., Gneiting, T. and Sloughter, J. M. (2009) EnsembleBMA: An $\mathrm{R}$ package for probabilistic forecasting using ensembles and Bayesian model averaging. Technical Report 516R, Department of Statistics, University of Washington. Available at: www.stat.washington.edu/research/reports/2008/tr516.pdf

Fraley, C., Raftery, A. E. and Gneiting, T. (2010) Calibrating multimodel forecast ensembles with exchangeable and missing members using Bayesian model averaging. Mon. Wea. Rev. 138, 190-202.

Fraley, C., Raftery, A. E., Gneiting, T., Sloughter, J. M. and Berrocal, V. J. (2011) Probabilistic weather forecasting in $\mathrm{R}$. The $R$ Journal 3 , 55-63. 
Gneiting, T., Larson, K., Westrick, K., Genton, M. G. and Aldrich, E. (2006) Calibrated probabilistic forecasting at the Stateline wind energy center: the regime-switching spacetime method. J. Amer. Stat. Assoc. 101, 968-979.

Gneiting, T. and Raftery, A. E. (2007) Strictly proper scoring rules, prediction and estimation. J. Amer. Statist. Assoc. 102, 359-378.

Gneiting, T. and Raftery, A. E. (2005) Weather forecasting with ensemble methods. Science 310, 248-249.

Gneiting, T., Raftery, A. E., Westveld, A. H. and Goldman, T. (2005) Calibrated probabilistic forecasting using ensemble model output statistics and minimum CRPS estimation. Mon. Wea. Rev. 133, 1098-1118.

Grimit, E. P. and Mass, C. F. (2002) Initial results of a mesoscale short-range ensemble forecasting system over the Pacific Northwest. Wea. Forecasting 17, 192-205.

Hágel, E. (2010) The quasi-operational LAMEPS system of the Hungarian Meteorological Service. Időjárás 114, 121-133.

Horányi, A, Kertész, S., Kullmann, L. and Radnóti, G. (2006) The ARPEGE/ALADIN mesoscale numerical modeling system and its application at the Hungarian Meteorological Service. Időjárás 110, 203-227.

Horányi, A., Mile, M., Szűcs, M. (2011) Latest developments around the ALADIN operational short-range ensemble prediction system in Hungary. Tellus A 63, 642-651.

Hyndman, R. J., Fan, Y. (1996) Sample quantiles in statistical packages. Amer. Statist. 50, 361-365.

Justus, C. G., Hargraves, W. R., Mikhail, A. and Graber, D. (1978) Methods for estimating wind speed frequency distributions. J. Appl. Meteor. 17, 350-353.

Lee, G. and Scott, C. (2012) EM algorithms for multivariate Gaussian mixture models with truncated and censored data. Comput. Statist. Data Anal. 56, 2816-2829.

Leith, C. E. (1974) Theoretical skill of Monte-Carlo forecasts. Mon. Wea. Rev. 102, 409-418.

Leutbecher, M. and Palmer, T. N. (2012) Ensemble forecasting. J. Comp. Phys. 227, 35153539.

McLachlan, G. J. and Krishnan, T. (1997) The EM Algorithm and Extensions. Wiley, New York.

Raftery, A. E., Gneiting, T., Balabdaoui, F. and Polakowski, M. (2005) Using Bayesian model averaging to calibrate forecast ensembles. Mon. Wea. Rev. 133, 1155-1174. 
Raftery, A. E., Kárný, M. and Ettler, P. (2010) Online prediction under model uncertainty via Dynamic Model Averaging: application to a cold rolling mill. Technometrics 52, 52-66.

Schuhen, N., Thorarinsdottir, T. L. and Gneiting, T. (2012) Ensemble model output statistics for wind vectors. Mon. Wea. Rev. 140, 3204-3219.

Sloughter, J. M., Gneiting, T. and Raftery, A. E. (2010) Probabilistic wind speed forecasting using ensembles and Bayesian model averaging. J. Amer. Stat. Assoc. 105, 25-37.

Sloughter, J. M., Gneiting, T. and Raftery, A. E. (2012) Probabilistic wind vector forecasting using ensembles and Bayesian model averaging. Mon. Wea. Rev. doi: 10.1175/MWR-D12-00002.1.

Sloughter, J. M., Raftery, A. E., Gneiting, T. and Fraley, C. (2007) Probabilistic quantitative precipitation forecasting using Bayesian model averaging. Mon. Wea. Rev. 135, 3209-3220.

Thorarinsdottir, T. L. and Gneiting, T. (2010) Probabilistic forecasts of wind speed: ensemble model output statistics by using heteroscedastic censored regression. J. Roy. Statist. Soc. Ser. A 173, 371-388.

Toth, Z. and Kalnay, E. (1997) Ensemble forecasting at NCEP and the breeding method. Mon. Wea. Rev. 125, 3297-3319.

Wilks, D. S. (2011) Statistical Methods in the Atmospheric Sciences. 3rd ed., Elsevier, Amsterdam. 